\section{Bringing on the BRAF}

\section{By Tracey Baas, Senior Editor}

The BRAF inhibitor Zelboraf vemurafenib from Daiichi Sankyo Co. Ltd. and Roche produces about an $80 \%$ response rate in its approved indication of BRAF-mutant melanoma but only about a 5\% rate in colorectal tumors with the same mutation. Now, European and U.S. teams have independently pinpointed epidermal growth factor receptor as the likely missing link in these colorectal cancers, suggesting treatments combining inhibitors of both targets could be more effective than monotherapy. ${ }^{1,2}$ Both groups are independently planning clinical trials with such combinations.

A group led by René Bernards, professor and head of molecular carcinogenesis at The Netherlands Cancer Institute, used an RNAibased genetic screen in human mutant colorectal cancer cells to identify kinases whose knockdown synergized with inhibition of the V600E mutant form of BRAF.

A team led by Jeffrey Engelman used phospho-kinase arrays to identify activation of kinase signaling during BRAF(V600E) inhibition in human colorectal cancer cells. Kinases phosphorylated by the arrays showed which pathways became activated to potentially circumvent BRAF inhibition. Engelman is director of the Center for Thoracic Cancers at the Massachusetts General Hospital Cancer Center and assistant professor of medicine at Harvard Medical School.

Both teams' experiments pointed to epidermal growth factor receptor (EGFR) as a contributor to BRAF resistance in colorectal cancer and thus as a target to restore the effects of BRAF inhibitors. Previous clinical trials of EGFR inhibitors alone have shown limited benefit in BRAFmutant colon cancer, which had led researchers to set their sights on targets other than EGFR. ${ }^{3-5}$

Bernards' group showed that BRAF-mutant colorectal cancer cell lines did not have a significant response to BRAF or EGFR inhibitors alone. BRAF inhibitor monotherapy also led to higher levels of phosphorylated EGFR than no treatment. Activated EGFR then renewed cell growth through the phosphoinositide 3-kinase (PI3K)-protein kinase B (PKB; PKBA; AKT; AKT1) pathway.

Cells treated with Zelboraf plus an EGFR inhibitor-Erbitux cetuximab, Iressa gefitinib or Tarceva erlotinib-showed greater apoptosis and less viability than cells treated with Zelboraf alone.

In colorectal cancer xenograft mice, a Zelboraf analog plus Erbitux or Tarceva decreased tumor growth compared with any of the molecules as monotherapy, suggesting the combination could help treat BRAFmutant cancers.
Data were published in Nature. The team included researchers from The Netherlands Cancer Institute, the University of Torino, the Italian Foundation for Cancer Research Institute of Molecular Oncology and the Catalan Institute of Oncology.

Engelman's group showed that Zelboraf plus Iressa led to sustained inhibition of MAPK (ERK), which sits downstream of EGFR, and decreased viability in BRAF-mutant colorectal cancer cell lines. The combination also led to tumor regression in xenograft mice. In contrast, Zelboraf alone led to incomplete suppression of ERK and renewed cell growth.

The team also studied patient biopsies and found that $60 \%$ of BRAF-mutant colorectal cancer samples had high levels of activated EGFR, whereas only $18 \%$ of BRAF-mutant melanoma biopsies had high levels of activated EGFR.

Results were published in Cancer Discovery. The team included researchers from Massachusetts General Hospital Cancer Center, Harvard Medical School, Tufts Medical Center, Mass a chuset t s General Hospital and Genentech Inc.

"The findings of the two
groups are potentially
groundbreaking,
demonstrating a novel
mechanism of intrinsic
resistance that is distinct
compared to the findings in
melanoma, thereby providing
a unique opportunity to
combine EGFR- and BRAF-
targeted agents in clinical
trials for BRAF-mutant
colorectal cancer."
-Jeffrey Legos,
GlaxoSmithKline plc

Zelboraf is marketed to treat metastatic melanoma. The drug is in Phase II testing for thyroid cancer, and a Phase I trial has been completed for colorectal cancers.

Tarceva is marketed by Astellas Pharma Inc., Chugai Pharmaceutical Co. Ltd., and Roche and its Genentech unit to treat non-small cell lung cancer (NSCLC) and pancreatic cancer. Erbitux, from Eli Lilly and Co., Bristol-Myers Squibb Co. and Merck KGaA, is marked to treat colorectal cancer and head and neck cancer and is under FDA review for NSCLC.

AstraZeneca plc's Iressa is marketed in the EU to treat NSCLC.

\section{Pathway to well}

Although both sets of results point to EGFR as a key mediator of resistance to BRAF, each group has its own idea about the mechanism by which EGFR leads to resistance and renewed cell growth (see Figure 1, "BRAF inhibition leads to EGFR-mediated pathway activation").

Bernards' group thinks BRAF inhibition causes increased levels of EGFR phosphorylation. Ultimately, this results in activation of the PI3K-AKT pathway, which renews cell proliferation.

Engelman's team hypothesized that resistance and renewed growth also could be due to EGFR-mediated reactivation of the Ras-Raf-MEKERK pathway. 


\section{Figure 1. BRAF \\ inhibition leads to \\ EGFR-mediated pathway activation. \\ Two groups suggest epidermal growth factor receptor (EGFR)-mediated pathway activation evades BRAF inhibi- tion. Each group has its own idea about the mechanism by which EGFR potentially mediates the pathway activation that leads to renewed cell growth.}

Prahallad et

al. think that in BRAF(V600E)-mutant cancers, normal

EGFR signaling results in activation of both the phosphoinositide 3-kinase (PI3K)-protein kinase B (PKB; PKBA; AKT; AKT1) pathway and the Ras-Raf-MEKMAPK (ERK) pathway. Cell division cycle 25C (CDC25C; EGFR activation (EGFR*). EGFR* then results in greater activation of the PI3K-AKT pathway (bold arrows in II).

Corcoran et al. think that in BRAF(V600E)-mutant cancers, EGFR ${ }^{\star}$ does not activate Ras, due to an ERK-dependent negative feedback loop-possibly via sprouty (SPRY) proteins (III). The group postulates that BRAF inhibition leads to feedback activation of Ras by EGFR, allowing EGFR to reactivate the Ras-Raf-MEK-ERK pathway (bold arrows in IV).

For either of the proposed EGFR-mediated mechanisms, EGFR inhibitors would potentially block pathway activation and subsequent renewed cell growth.

Regardless of the precise mechanism, both teams' findings provide a rationale for the poor clinical response of BRAF(V600E)-mutant colorectal cancers to BRAF monotherapy. The data also suggest EGFR levels might be a useful biomarker to decide whether to use monotherapy or combination therapy for any cancer harboring the BRAF(V600E) mutation.

"The preclinical data are very strong, and it is quite feasible to move into clinical trials," said Engelman. "Because BRAF and EGFR inhibitors are already FDA approved and two separate labs have seen the same effects, I think the translation to clinical trials will be pretty straightforward."

"The interesting thing is that even though EGFR inhibitors alone have shown no clinical benefit in patients with BRAF(V600E)-mutant
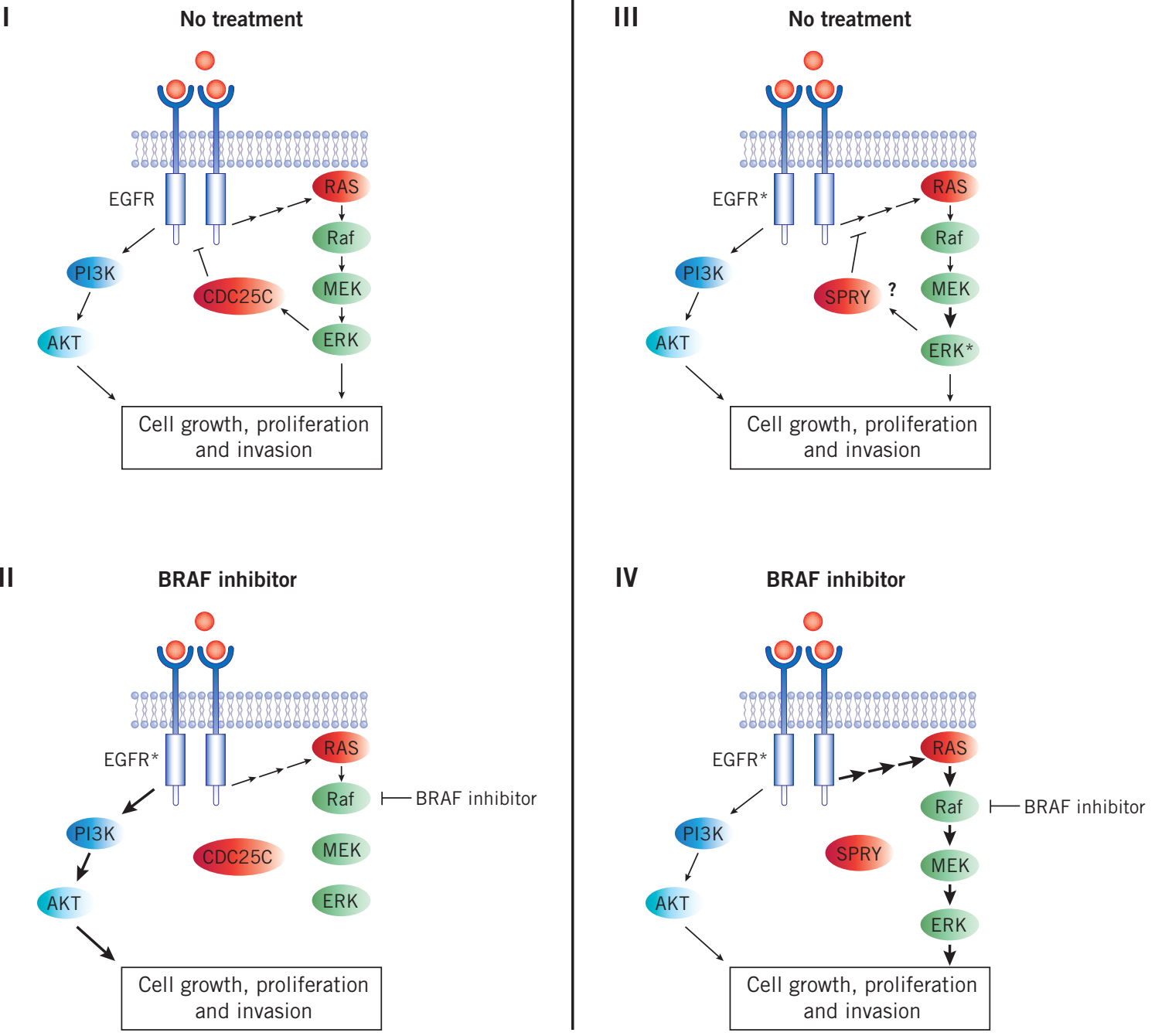
HER2-positive metastatic breast cancer and is also in ongoing Phase III trials in gastric cancer and head and neck cancer.

"And although the groups each have their own interesting point of view on the EGFR-mediated mechanism, their common findings give a possible explanation as to why monotherapy had been a bit dismal for BRAF-mutant colorectal cancer, compared to what we

\section{"As to what this could mean for programs at Genentech, the immediate implications would be the potential opportunity to test EGFR inhibitors in combination with other inhibitors aimed at the Ras-Raf-MEK-ERK pathway." - Jeffrey Settleman, Genentech Inc.} would have expected data for this class of drug," added Legos. "Engelman's EGFR characterization of patient biopsies gives further credibility, bridging what is occurring in the clinic to what was determined in the based on melanoma laboratory."

"The work provides a preclinical rationale, in a specific context, for BRAF inhibitors to provide benefit in combination with EGFR inhibitors-which is a combination that would not have been obvious based on prior studies of these pathways," Jeffrey Settleman told SciBX.

Settleman, a coauthor on the Cancer Discovery manuscript, is senior director of the discovery oncology program at Genentech. Previously, he was professor of medicine at Massachusetts General Hospital.

"As to what this could mean for programs at Genentech, the immediate implications would be the potential opportunity to test EGFR inhibitors in combination with other inhibitors aimed at the Ras-Raf-MEK-ERK pathway," Settleman said.

Genentech's MEHD7945A, a humanized IgG1 mAb targeting EGFR and EGFR3 (HER3; ERBB3), is in Phase I testing for metastatic epithelial tumors.

GDC-0973, a MEK inhibitor from Genentech, Roche and Exelixis Inc., is in Phase I trials to treat melanoma and solid tumors.

"The more far-reaching implications would be the need to more fully understand inhibitor-driven pathway feedback and the redundancy in that feedback," said Settleman. "When one pathway is blocked, what pathway comes up to compensate for that blockade? Screening strategies, such as those using siRNA or gain of function, are going to help researchers uncover the interplay between different pathways that lead to resistance and more importantly suggest potential target combinations to circumvent that resistance."

Both Bernards' and Engelman's work is unpatented, and both researchers are in discussions with undisclosed parties to start clinical trials of BRAF and EGFR inhibitors to treat mutant colorectal cancers

Baas, T. SciBX 5(6); doi:10.1038/scibx.2012.140

Published online Feb. 9, 2012

\section{REFERENCES}

1. Prahallad, A. et al. Nature; published online Jan. 26, 2012; doi:10.1038/nature10868

Contact: René Bernards, The Netherlands Cancer Institute, Amsterdam, the Netherlands e-mail: r.bernards@nki.nl

2. Corcoran, R.B. et al. Cancer Discov.; published online Jan. 16, 2012; doi:10.1158/2159-8290.CD-11-0341

Contact: Jeffrey A. Engelman, Massachusetts General Hospital Cancer Center, Boston, Mass. e-mail: jengelman@partners.org

3. Di Nicolantonio, F. et al. J. Clin. Oncol. 26, 5705-5712 (2008)

4. Karapetis, C.S. et al. N. Engl. J. Med. 359, 1757-1765 (2008)

5. Amado, R.G. et al. J. Clin. Oncol. 26, 1626-1634 (2008)

\section{COMPANIES AND INSTITUTIONS MENTIONED}

Astellas Pharma Inc. (Tokyo:4503), Tokyo, Japan

AstraZeneca plc (LSE:AZN; NYSE:AZN), London, U.K.

Bristol-Myers Squibb Co. (NYSE:BMY), New York, N.Y. Catalan Institute of Oncology, L'Hospitalet-Barcelona, Spain Chugai Pharmaceutical Co. Ltd. (Tokyo:4519), Tokyo, Japan Daiichi Sankyo Co. Ltd. (Tokyo:4568; Osaka:4568), Tokyo, Japan Eli Lilly and Co. (NYSE:LLY), Indianapolis, Ind.

Exelixis Inc. (NASDAQ:EXEL), South San Francisco, Calif. Genentech Inc., South San Francisco, Calif.

GlaxoSmithKline plc (LSE:GSK; NYSE:GSK), London, U.K

Harvard Medical School, Boston, Mass.

Italian Foundation for Cancer Research Institute of Molecular

Oncology, Milan, Italy

Massachusetts General Hospital, Boston, Mass.

Massachusetts General Hospital Cancer Center, Boston, Mass. Merck KGaA (Xetra:MRK), Darmstadt, Germany

The Netherlands Cancer Institute, Amsterdam, the Netherlands Roche (SIX:ROG; OTCQX:RHHBY), Basel, Switzerland

Tufts Medical Center, Boston, Mass.

University of Torino, Torino, Italy 